\title{
Research on Modeling and Simulation of the anti-submarine patrol aircraft taking call search task using the circular sonar buoy array
}

\author{
Ma Zheng-wei ${ }^{1, *}$, Long Fei ${ }^{1}$, and Zhao Xin $\mathrm{Ye}^{1}$ \\ ${ }^{1}$ Dalian Navy Academy, Dalian, China
}

\begin{abstract}
According to the characteristics of the call search task, this paper analyzes the submarine position dispersion model, and puts forward the evaluation model of the submarine buoy array search probability based on the method of the circular sonar buoy array. The correctness of the model is proved by the computer simulation method, and the results show that the results of the submarine search are closely related to the submarine position, and the method can provide a useful reference for the optimal use of sonar array.
\end{abstract}

\section{Introduction}

Compared with the helicopter, the anti-submarine patrol has the advantages of high speed, long range, long life time, and a variety of anti-submarine combat equipment on board. Therefore, it becomes an important aviation anti-submarine force.

Call anti-submarine warfare is one of the most basic and most commonly used methods. It refers to the antisubmarine patrol aircraft at the airport or designated airspace, flies to the initial position of the submarine. After obtaining information about the activities of the enemy submarine, then takes combat operations about searching, locating, tracking and attacking submarine.

In order to accomplish this task, some submarine detection equipment is needed, such as radar, photoelectric equipment, radio sonobuoy, mad, etc. Among them, the radio sonobuoy is a good and effective equipment, and it is subject to geographical constraints, more use of convenient exploration, so it attaches great importance by navies in most countries.

\section{A distributed model of submarine position for call search}

\subsection{The initial position of the submarine during the call search}

Due to the uncertainty of the submarine position data from other sources, according to the central limit theorem, it can be considered that the initial position of the submarine obeys two-dimensional normal distribution $N\left(0, \sigma_{0}{ }^{2}\right)$, and the distribution center is at

\footnotetext{
Corresponding author: kongshuguang88@sina.com
}

the initial position (coordinate origin), that is, the mathematical expectation value is 0 , and the joint probability density function of the initial position (X, Y) is

$$
f(x, y)=\frac{1}{2 \pi \sigma_{0 x} \sigma_{0 y}} e^{-\left[\frac{x^{2}}{2 \sigma_{0 x}{ }^{2}}+\frac{y^{2}}{2 \sigma_{0 y}{ }^{2}}\right]}
$$

Among them, $x$ and $y$, are independent and identically distributed, when $\sigma_{0 x}=\sigma_{0 y}=\sigma_{0}$, and the joint density function is obtained by the transformation from Cartesian coordinates to polar coordinates:

$$
\varphi(r, \theta)=f(r \cos \theta, r \sin \theta)\left|\frac{\partial(x, y)}{\partial(r, \theta)}\right|=\frac{r}{2 \pi \sigma_{0}^{2}} e^{-\frac{r 2}{2 \sigma_{0}^{2}}},(r, \theta) \in D
$$

Where, $D=\{(r, \theta) \mid r>0, \theta \in[0,2 \pi]\}$ 。

\subsection{Location dispersion of submarine after call search}

In the time, which is from the initial information of the submarine position to the anti-submarine force arrives at the search area, the position of the submarine continues to expand. It is centered on the initial position, and the speed of the original navigation

The size of the dispersion area is related to the speed and the dominant time of the submarine (the call time)Therefore, the current position dispersion of the submarine should contain two parts: the initial dispersion and the uncertainty caused by the motion。

In most cases, the speed of the submarine is unknown. When the submarine speed is unknown and the course 
obeys uniform distribution in $[0,2 \pi]$. When the antisubmarine forces begin to search after the call time $t_{0}$, the position of the submarine is distributed due to the uncertainty of submarine velocity.

The position distribution caused by submarine motion obeys normal distribution $N\left(0, \sigma_{1}^{2}\right)$, because of the deviation from the center position $\left(x_{1}, y_{1}\right)$ caused by the submarine movement,

The error of the initial position information of submarine is normally distributed $N\left(0, \sigma_{0}^{2}\right)$, and the position of the submarine deviates from the center

$\left(x_{0}, y_{0}\right)$ because of the error of the information source, At the beginning of the sonar array, the initial position of the submarine is in $\left(x_{0}+x_{1}, y_{0}+y_{1}\right)$ 。

Because $\left(x_{0}, y_{0}\right)$ and $\left(x_{1}, y_{1}\right)$ are independent and identically distributed, it is not difficult to prove that the normal distribution $\left(x_{0}+x_{1}, y_{0}+y_{1}\right)$ obeys normal distribution $N\left(0, \sigma_{0}^{2}+\sigma_{1}^{2}\right)$. According to the formula (2), in the polar coordinates, the density function of submarine position is:

$$
\varphi(r, \theta)=\frac{r}{2 \pi\left(\sigma_{0}^{2}+\sigma_{1}^{2}\right)} e^{-\frac{r^{2}}{2\left(\sigma_{0}^{2}+\sigma_{1}^{2}\right)}}
$$

Where, the $\sigma_{1}^{2}$ is still unknown, and the velocity distribution law of submarine is analyzed as following, in order to solve the $\sigma_{1}^{2}$ value:

The position probability density function of the submarine, caused by the uncertainty of velocity and course, can be expressed in polar coordinates as following:

$$
\begin{aligned}
& \varphi_{R}(r)=\int_{0}^{2 \pi} \varphi(r, \theta) d \theta=\frac{r}{\sigma_{0}^{2}} e^{-\frac{r^{2}}{2 \sigma_{0}{ }^{2}}} I_{(0,+\infty)} \\
& \varphi_{w}(\theta)=\int_{0}^{+\infty} \varphi(r, \theta) d r=\frac{1}{2 \pi} I_{[0,2 \pi)}
\end{aligned}
$$

It can be seen from the formula $\varphi(r, \theta)=\varphi_{R}(r) \varphi_{w}(\theta)$, that in polar coordinates, $r 、 \theta$ is independent, $r$ obeys Rayleigh distribution, and $\theta$ obeys the uniform distribution on the interval. From the formula (4), we can obtain the probability density function of submarine speed $\mathrm{V}$ :

$$
\varphi(\mathrm{v})=\varphi_{R}(\mathrm{v})\left|\frac{\mathrm{dr}}{\mathrm{dv}}\right|=\frac{v t_{0}{ }^{2}}{\sigma_{1}^{2}} e^{-\frac{r^{2}}{2 \sigma_{1}^{2}}} \boldsymbol{I}_{(0,+\infty)}
$$

If $\sigma_{1}=\sigma_{v} t_{0}$, then the upper form can be expressed as following:

$$
\varphi(\mathrm{v})=\frac{v}{\sigma_{v}^{2}} e^{-\frac{v^{2}}{2 \sigma_{v}^{2}}} I_{(0,+\infty)}
$$

The economic speed of the submarine $V_{s e}$ is taken as the mean of the velocity $\mathrm{V}$ distribution function, according to the definition of mean:

$$
E[v]=V_{s e}=\int_{0}^{+\infty} v \varphi(\mathrm{v}) \mathrm{dv}=\sqrt{\frac{\pi}{2}} \sigma_{v},
$$

Thus we can get: $\sigma_{1}=\sigma_{1} t_{0}=\sqrt{\frac{2}{\pi}} V_{s e} t_{0}$

So far, the probability density function of the submarine position is obtained when the anti-submarine patrol machine starts to search through time $t_{0}$ :

$$
\left\{\begin{array}{l}
\varphi(r, \theta)=\frac{r}{2 \pi \sigma^{2}} e^{-\frac{r^{2}}{2 \sigma^{2}}} \\
\sigma^{2}=\sigma_{0}^{2}+\frac{2}{\pi}\left(V_{s e} t_{0}\right)^{2}
\end{array}\right.
$$

\section{Deployment Circular sonar array to call search the submarine}

In the anti-submarine patrol aircraft taking call the search task, for the situation about known the initial target location, it placed a sonar buoy in the general dispersion center as soon as possible, in order to shorten the time of the submarine was found for the first time, and then deploy some sonar buoys around the center by a circular array, and try to cover a large area as large as possible.

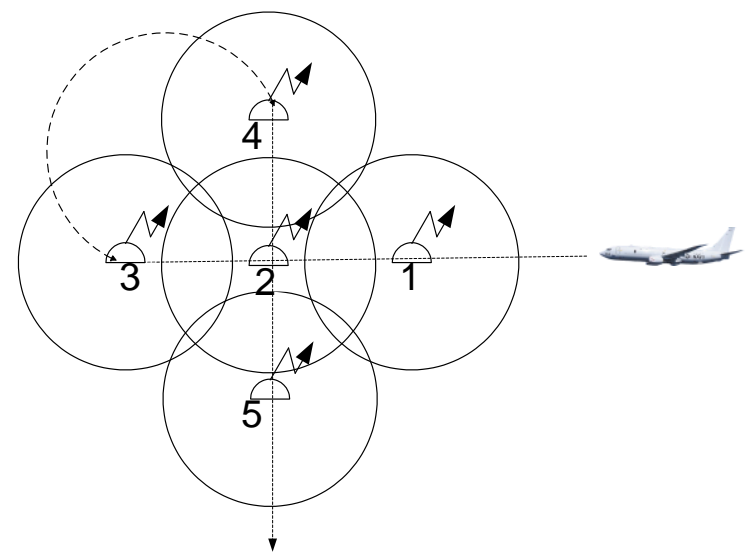

Fig. 1 Schematic diagram of the typical 5 circular search arrays 


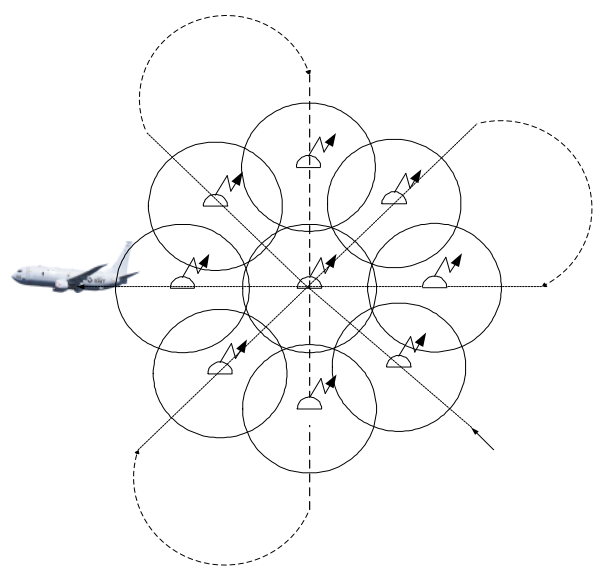

Fig. 2 Schematic diagram of the typical 9 circular search arrays

Considering that there is little difference between the circular and annular array in the actual operation (the main difference is that the annular array can be omitted from the center point buoy), the search efficiency of the call anti-submarine warfare is considered according to the circular array.

When determining the number of buoys to be used, it should be considered that there is a certain spread of each sonar buoy. In order to ensure reliable detection, the distance between two sonar buoys should be less than 2 times buoy's range. The size of the sonar buoys array is related to some elements, such as lost time which the anti- submarine patrol aircraft and submarine formation lost contact (call time), the submarine speed, the number of sonar buoy on the plane, and so on. The typical circular (or annular) search array has 5, 7, 9 or 11 sonar buoys, and 5 and 7 circular search arrays are as shown in Figure 1 and Figure 2.

The flight path is depended on both the distance between two sonar buoy and the plane turning radius. And the deploying order is as much as possible to make the flight route optimization, that is, deploying array time should be short, and the aircraft's large slope turn and maneuver should be less.

Taking 5 passive omnidirectional sonar buoy circular array for example, the deployment sonar buoys process is illustrated as following:

When the probe range of sonar buoy is $3.8 \mathrm{~km}$ and the interval between the buoys is $7 \mathrm{~km}$, the first buoy should be layed out in the position deviate from the submarine initial point $5 \mathrm{~km}$, and then throw second buoys after fly before $5 \mathrm{~km}$, then the third also after fly ahead $5 \mathrm{~km}$;

After the aircraft to turn left at the $5 \mathrm{~km}$ radius, when flying through the fourth buoy locations above, it throws the buoy; when the direction of flight and the original direction of 90 degrees and from the first buoys to $5 \mathrm{~km}$, throw the fifth buoys.

The plane flies almost in a great circle in the whole laying array process (see Figure 1), it is mainly due to the aircraft turning limit, and this is one of maneuvering performance difference between the large and medium sized fixed wing anti-submarine patrol aircraft and antisubmarine helicopter. According to the performance of each type of aircraft, there are differences in the corresponding turning radius. For the modern antisubmarine patrol aircraft, when anti-submarine patrol aircraft's command and control system will outputs the flight path of the array for Tactical Navigation System, flight control system can automatically control the plane array process.

\section{Efficiency model and Simulation of call search}

\section{1 call search probability}

Assuming that the moving velocity $V_{s e}$ of the target obeys the Rayleigh distribution, the course obeys the uniformly distributed in the interval $[0,2 \pi]$, The initial position of the target obeys the normal distribution $N\left(0, \sigma_{0}{ }^{2}\right)$. It throws sonar buoy in the place with initial position as the center, and the action distance of the sonar buoy is $R_{f}$, After the time $t_{0}$, the probability of call search submarine as following:

$$
\begin{gathered}
P S=\iint_{\substack{r \leq R_{f} \\
0 \leq \theta<2 \pi}} \frac{r}{2 \pi \sigma^{2}} e^{-\frac{r^{2}}{2 \sigma^{2}}} d r d \theta=1-e^{-\frac{r^{2}}{2 \sigma^{2}}} \\
\text { Where: } \sigma^{2}=\sigma_{0}^{2}+\frac{2}{\pi}\left(V_{s e} t_{0}\right)^{2}
\end{gathered}
$$

\subsection{Model validation}

Assuming that: the initial distribution of the target is 1 nautical miles (mean square error) when in call search submarine, and the speed of the anti-submarine patrol aircraft is about $400 \mathrm{~km} / \mathrm{h}$. The detected range of a single passive sonar buoy is calculated according to the hydrological conditions of the search area

Because the single sonar buoy's detection range is limited, in the actual aviation anti-submarine search action, it usually lays a set of sonar buoys to form a search array, which is in accordance with the spread law of submarine's position, and cover the search area as much as possible. In this paper, the simulation verification is based on 9 round sonar buoy array.

First of all, according to the submarine distribution law as shown in chapter 1.2 , and combination with the characteristics of the search task, the plane lays out a circular search array composed of multiple buoys. For simulation of the search process in computer, the specific steps are as follows:

(1) Input the initial condition. Including the submarine speed (0-24kn), each buoy's coordinate parameter which composed of search array.

(2) Generate the initial position of the target, when in call search task. 
(3) When the target's speed is unknown, generate the target's velocity obeying the Rayleigh distribution, based on the average speed of the submarine $V_{\text {se }}$.

(4) Produce the target course, obeying the Uniformly distributed in the interval $[0,2 \pi]$.

(5) Put down the sonar buoy and calculate the submarine position. In the meantime, the calculation point can be added in the process of putting the sonar buoy, in order to improve the simulation precision.

(6) Determine whether the set of equations is conect.

$$
\left\{\begin{array}{c}
\left(\mathrm{x}-\mathrm{a}_{1}\right)^{2}+\left(\mathrm{y}-\mathrm{b}_{1}\right)^{2} \leq \mathrm{R}_{\mathrm{f}}{ }^{2} \\
\left(\mathrm{x}-\mathrm{a}_{2}\right)^{2}+\left(\mathrm{y}-\mathrm{b}_{2}\right)^{2} \leq \mathrm{R}_{\mathrm{f}}{ }^{2} \\
\cdots \cdots \cdots \cdots \\
\left(\mathrm{x}-\mathrm{a}_{\mathrm{i}}\right)^{2}+\left(\mathrm{y}-\mathrm{b}_{\mathrm{i}}\right)^{2} \leq \mathrm{R}_{\mathrm{f}}{ }^{2}
\end{array}\right.
$$

Where, $\left(a_{i}, b_{i}\right)$ is the position coordinates of each buoy in buoy array, $R_{f}$ is the detection range of the sonar buoy.

(7) When any set of equations in the equation is set up, it means getting the target once, and increase the count by one. Then begin the next cycle continue by (1)(7), until the maximum number of cycles.

(8) at last, statistical the search probability.

Then, in the same search conditions both the submarine state and the formation of sonar buoy array, it calculates the probability of the call search task, using the search evaluation model subscribed in formula (6).

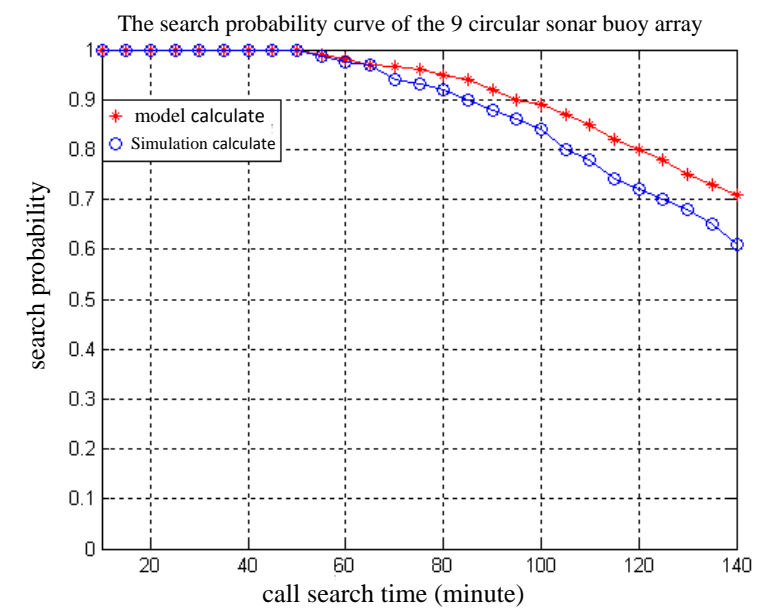

Figure. 3 The search probability curve of the 9 circular sonar buoy array for the unknown submarine

Figure 3 shows the results of the evaluation of the search probability for the circular array of the 9 sonar buoys, using the formula of search probability and the method of computer simulation. The result is shown in figure 3, that the curve with cross mark is the result of the model formula, and the curve of diamond mark is the result of computer simulation.

As is shown in Figure 3, the result of the model calculating is consistent with the simulation results when the anti-submarine patrol machine performs the call search task.

In order to make the calculation results of the model approximate to the simulation results, the corresponding correction coefficients can be added to the model formula. The corrected results are shown in Figure 4. And the two curves in the figure are basically coincident.

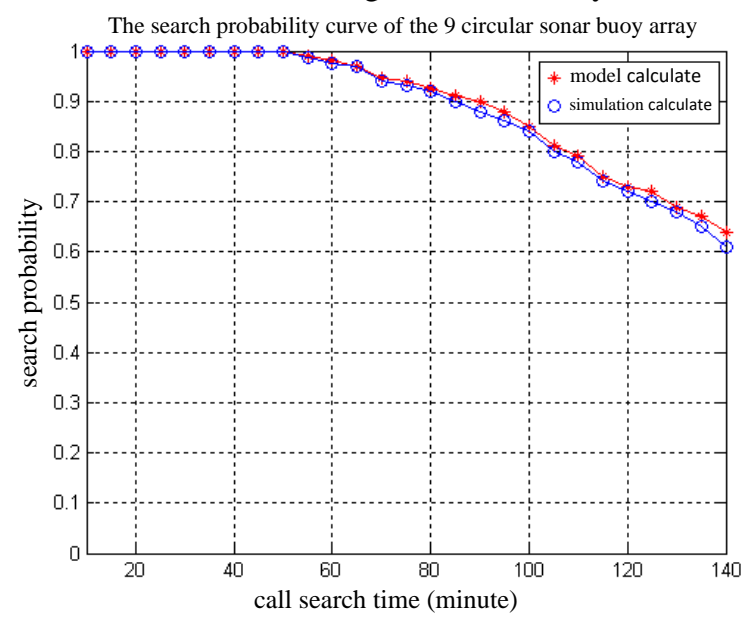

Figure. 4 The search probability curve of the modified 9 circular sonar buoy array for the unknown submarine

\section{Conculsion}

The efficiency calculation of the search for the sonar buoy array is a complicated problem,

The paper established the calculation model of circular sonar buoy array searching submarine, using the proposed submarine position distribution model. The result of respectively assessment model calculation and computer simulation method for searching probability calculation is consistent, which proves the correctness of the model. It also shows that the evaluation results of submarine is closely related to the submarine position distribution, and it should be selected reasonably according to the situation. In the practical use, the correction term can be added to the model, which makes the calculation results of the model and the simulation results closer.

\section{Acknowledgements}

Acknowledgement: this work is supported by the Dalian Navy Academy Research Development Foundation Academic Topic (2016030).

\section{REFERENCES}

1. Sun Mingtai. Aviation Antisubmarine tactics[M]. Beijing: Military Science Press, 2003;

2. Qu Yepin. Search effectiveness evaluation and decision modeling of anti-submarine[M]. National Defence Industry Press, 2011

3. Wan Xinwei, Tan Ansheng. Summarize research on the anti-submarine patrol aircraft [J].Military 
Operations Research and Systems Engineering, 2015.12, 29 (4) : 72-78.

4. Cai Yunxiang, Chu lei, Gong Xu. ASW-aircraft Anti-submarine Orientating Error for Factor Effection with Buoy[J]. Ship Electronic Engineering, 2016.06, 36 (6) : 38-40.

5. Sun Huachun,Li Changwen. Probability Model of Antisubmarine Helicopter Radar in Searching[J]. Ship Electronic Engineering, 2008,28(9):116-118.

6. LIU Yan-feng, PAN Quan, DU Zicheng.Coordinate System Problems in Airborne Radar Searching and Tracking[J]. Fire Control and Command Control,2005,30(3):40-43. 